\title{
Simulation and Design of a Submicron Ultrafast Plasmonic Switch Based on Nonlinear Doped Silicon MIM Waveguide
}

\author{
Ahmad Naseri Taheri, Hassan Kaatuzian \\ Photonics Research Laboratory (PRL), Electrical Eng. Dept, Amirkabir University of Technology, Tehran, Iran. \\ Email: ahmadnaseri@aut.ac.ir, hsnkato@aut.ac.ir
}

Received September 2013

\begin{abstract}
We propose and analyze a submicron stub-assisted ultrafast all-optical plasmonic switch based on nonlinear MIM waveguide. It is constructed by two silicon stub filters sandwiched by silver cladding. The signal wavelength is assumed to be $1550 \mathrm{~nm}$. The simulation results show a $-14.66 \mathrm{~dB}$ extinction ratio. Downscaling the silicon waveguide in MIM structure leads to enhancement of the effective Kerr nonlinearity due to tight mode confinement. Also, using $\mathrm{O}^{+}$ions implanted into silicon, the switching time less than $10 \mathrm{ps}$ and a delay time less than $8 \mathrm{fs}$ are achieved. The overall length of the switch is $550 \mathrm{~nm}$.
\end{abstract}

Keywords: Plasmonics; Silicon Based All-Optical Switch; Stub Filter; Metal-Insulator-Metal Waveguide; Nonlinear Kerr Effect

\section{Introduction}

Plasmonics promises the speed of light in nano-scale integration to the future of data processing and communications $[1,2]$. The combined advantages of ultrafast optical signal processing capabilities of photonics and the feasibility of nano-scale fabrication motivate researchers to work on Plasmonics [3-5]. In the past years, many techniques and devices in the field of Plasmonics are developed. Among those, Metal-Insulator-Metal (MIM) plasmonic waveguides provided many applications, because their mode confinement in deep subwavelength scale and high group velocity extended over wide range of frequencies, from DC to visible. Several applications of MIM plasmonic waveguides such as filters, Bragg Reflectors, modulators, and switches are reported [6-9]. In some of reports, remarkable properties of nonlinear Surface Plasmon Polariton: (SPP)-based structures are utilized to propose nanophotonic devices $[8,10]$. In order to eliminate the limit in switching speed due to electronics, all-optical methods are developed [11,12]. In most of the all-optical switching methods, a pump signal alters the refractive index of the path of another signal and changes its phase or intensity [12]. However, because of weak nonlinear properties of medium, they need more powerful pump and/or longer nonlinear waveguide for more effective interaction. In addition, the separation of data signal from pump signal requires an external filter in which increases the overall length of the switch. Stub structures have been used as wavelength selectivefilter in plas monic waveguides [13,14]. A stub has goofiltering effect for dis crete wavelengths.

In this paper, we propose an all-optical switch based on Metal-Insulator-Metal plasmonic structure and utilized two stub filters, one as active medium and the other as output filter. Silver-doped silicon is used as active material, in which reported to have giant Kerr nonlinearity $n_{2}=1.47 \times 10^{-9} \mathrm{~m}^{2} / \mathrm{W}$ [15]. We have used a periodic stub as Bragg reflector in order to filter control signal from data signal. Also, we have employed another periodic stub filter as an active region of our switch. Changing the refractive index of this periodic stub converts it into $1550 \mathrm{~nm}$ filter. The overall length of our switch is $550 \mathrm{~nm}$. Our design has numerically investigated based upon 2-D Finite Element Method simulations using COMSOL Multiphysics. In Section 2, we'll describe in brief, how a MIM plasmonic waveguide works. In Section 3, nonlinear Kerr effect in our proposed stub structure and its switching applications will be explained. In Section 4, we'll present the simulation results obtained in our plasmonic switch design. We'll also have a conclusion section.

\section{MIM Plasmonic Waveguide}

In spite of the severe loss, among the other schemes of 
plasmonic waveguides, MIM has the advantage of strong modal confinement in its subwavelength size. In this structure, two SPP's couple into the central dielectric slot and thus gives rise to a huge field concentration.

Figure 1 shows a schematic diagram of a Metal-Insulator-Metal waveguide. In this type of plasmonic waveguide a dielectric core is surrounded by two metal claddings. The dispersion relation of this waveguide described by [6]:

$$
\frac{k_{m}}{\varepsilon_{m}}=-\frac{k_{d}}{\varepsilon_{m}} \tanh \left(\frac{t}{2} k_{d}\right)
$$

Where $\varepsilon_{d}$ dielectric constant of dielectric core, $\varepsilon_{m}$ the dielectric function of metal cladding, and $k_{d}$ and $k_{m}$ are the transverse wave number in the core and the cladding, respectively. We use a seven-pole Drude-Lorentz model, in which is defined in the wavelength range from 0.2 to $2 \mu \mathrm{m}[16]$ :

$$
\varepsilon_{2}(\omega)=1-\frac{\omega_{p}^{2}}{\omega(\omega+i \gamma)}+\sum_{n=1}^{5} \frac{f_{n} \omega_{n}^{2}}{\omega_{n}^{2}-\omega^{2}-i \omega \gamma n}
$$

Where, $\omega_{p}=2002.6 \mathrm{THz}$ is the bulk plasma frequency of silver and $\gamma=11.61 \mathrm{THz}$ is a damping constant. Other parameters are listed in the Table 1. In all our simulations we applied the above model to create more accurate results.

\section{Kerr Effect in Stub Structure}

One of the common elements between microwave engineering and photonics is the stub structure which is utilized as wavelength selective filter. Many reports numerically demonstrate stub structures as compact size and simple filter [14]. A stub filter consists of one or more finite length waveguide(s) perpendicular to the main waveguide.

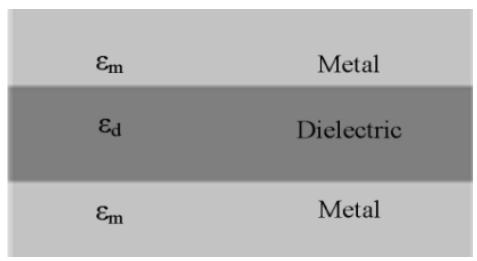

Figure 1. Schematic diagram of a metal-insulator-metal waveguide.

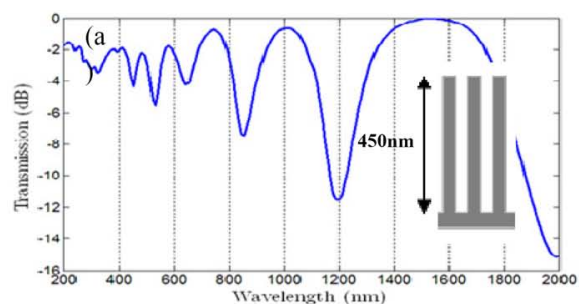

In our switch, we have employed two different stub filters, at input and output. Both of them have been separately simulated to obtain their transmission spectrum. Changing three parameters (mentioned in Table 1), could change the spectrum of the filter, the length of stub(s), the width of stub(s) and/or the refractive index of the core. For changing the transmission characteristics of this Silicon based plasmonic switch in "ON" and "OFF" states, we propose to use Silver-doped Silicon. So that nonlinear optical Kerr effect may be observed during $1550 \mathrm{~nm}$ signal switching using $1000 \mathrm{~nm}$ pump. Kerr occurs, when the $\mathrm{Si}$ waveguide is subjected to a strong pump field (E). It becomes doubly refracting. So the refractive index will be changed as follows $[17,18]$ :

$$
\Delta n=n_{2} I
$$

Utilizing nonlinear optical Kerr effect in silicon (the core of MIM waveguide and stub(s)), and based on femtosecond optical perturbation, we can change the refractive index of the MIM waveguide and therefore by shifting the transmission spectrum of the stub at $1550 \mathrm{~nm}$, the switching process would be achieved.

Figures 2(a) and (b) show the transmission of two filters vs. wavelength. The input stub filter should pass $1550 \mathrm{~nm}$ signal and $1000 \mathrm{~nm}$ pump. Also, by reasonable small changing the refractive index, this filter attenuates $1550 \mathrm{~nm}$ signal. Therefore, the length of stubs of this filter is selected $L_{s 1}=450 \mathrm{~nm}$.

The second stub, as shown in Figure 2(b), acts statically and only filters the $1000 \mathrm{~nm}$ pump signal. The length of its stubs is chosen so that it passes the $1550 \mathrm{~nm}$ signal and reflects the $1000 \mathrm{~nm}$ pump. Therefore, based on simulations we have chosen the length of this stub filter $L_{s 2}=200 \mathrm{~nm}$.

Table 1. Parameters Of The Drude-Lorentz Model For Silver [16].

\begin{tabular}{cccc}
\hline $\boldsymbol{n}$ & $\boldsymbol{\omega}_{\boldsymbol{n}}(\mathbf{T H z})$ & $\gamma_{\boldsymbol{n}}(\mathbf{T H z})$ & $\boldsymbol{f}_{\boldsymbol{n}}$ \\
\hline $\mathbf{1}$ & 197.3 & 939.62 & 7.9247 \\
$\mathbf{2}$ & 1083.5 & 109.29 & 0.5013 \\
$\mathbf{3}$ & 1979.1 & 15.71 & 0.0133 \\
$\mathbf{4}$ & 4392.5 & 221.49 & 0.8266 \\
$\mathbf{5}$ & 9812.1 & 584.91 & 1.1133 \\
\hline
\end{tabular}

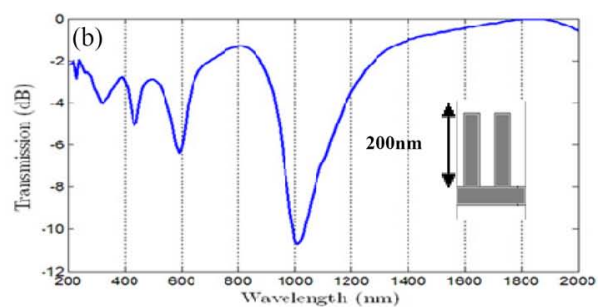

Figure 2. Transmission spectrum of (a) input filter with $400 \mathrm{n}$, stub Length and (b) output filter with $2000 \mathrm{~nm}$ stub length. 
The number of stubs in each filter depends on their functionality. In the first filter, we need to increase the effective length of the nonlinear region. So, three stubs design is proposed. But in second filter, two stubs are enough to reject the control beam from output.

\section{Plasmonic Switch Simulation Results}

Figure 3 depicts the proposed MIM plasmonic all-optical switch. It consists of a silicon waveguide sandwiched by silver.

The width of main waveguide and stubs of two filters is selected $\mathrm{W}=50 \mathrm{~nm}$ and the length of stubs of the input filter is $L_{s 1}=450 \mathrm{~nm}$ and the output filter $L_{s 2}=200 \mathrm{~nm}$ and the distance of them is $d=100 \mathrm{~nm}$. The overall length of the switch is $L=550 \mathrm{~nm}$. That means, our switch has subwavelength dimension. Figures 4(a)-(c) demonstrate the field distribution in the switch. As seen in Figures 4(a) and (c), in on-state the $1550 \mathrm{~nm}$ signal passes both filters and $1000 \mathrm{~nm}$ pump passes the input filter and changes its refractive index and reflected by second one. In off-state, after modifying refractive index of first filter, the $1550 \mathrm{~nm}$ signal is filtered and reflected to the input (Figure 4(b)). The overall transmission of the switch in $\mathrm{ON}$ and OFF state is shown in Figure 5. Imposing the control signal, the stop band of the control pulse (1000 $\mathrm{nm}$ ) is almost remained unchanged; however, the transmission of the switch falls at $1550 \mathrm{~nm}$.

Ion-Implanted Silicon (II-Si) helps the switching process in ultrafast sweeping the excited free-carriers in less than 10 fs $[11,12]$. Simulations show an 8 fs delay time for the

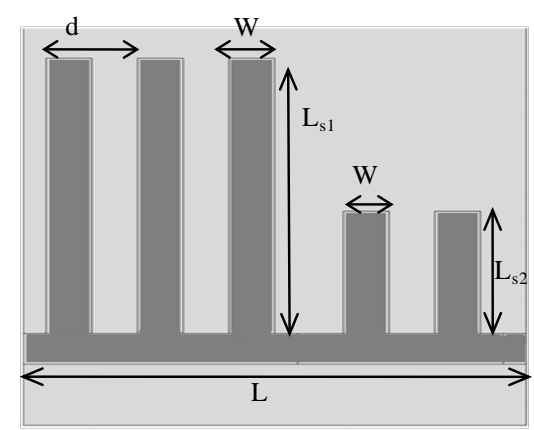

Figure 3. The proposed structure utilizing two stub filters based on MIM plasmonic waveguide.
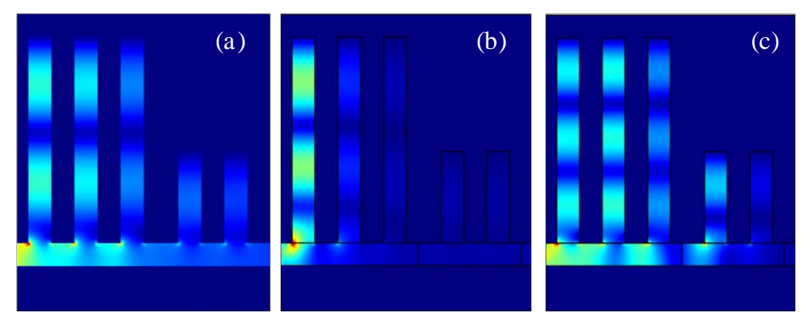

Figure 4. Intensity distribution of (a) $1550 \mathrm{~nm}$ at “on” state (b) $1550 \mathrm{~nm}$ at “off” state (c) $1000 \mathrm{~nm}$ as pump signal. optical pulse to pass the switch (Figure 6). Figure 7 depicts the output power of $1550 \mathrm{~nm}$ signal respect to power of $1000 \mathrm{~nm}$ pump power. The extinction ratio is calculated $-14.66 \mathrm{~dB}$.

\section{Conclusion}

In this paper, the all-optical plasmonic switch based on metal-insulator-metal structure is designed and simulated. In the structure of the switch, two stub filters are embedded. These filters are designed and analyzed to perform with the communication wavelength $1550 \mathrm{~nm}$. The small

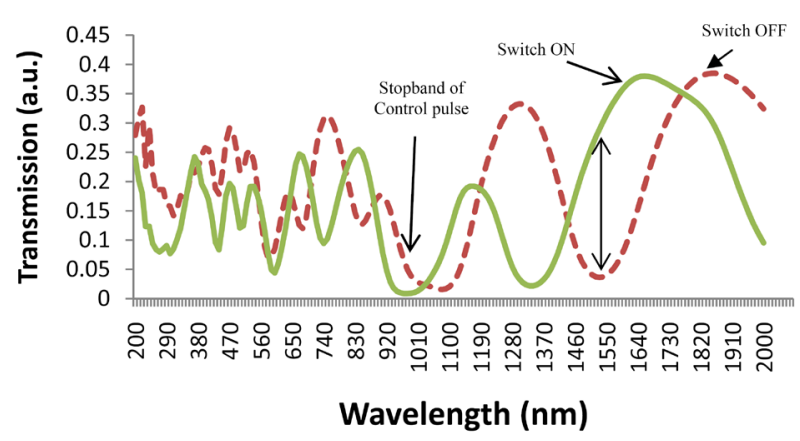

Figure 5. The overall transmission of the switch in OFF (dashed) and ON (solid) state.

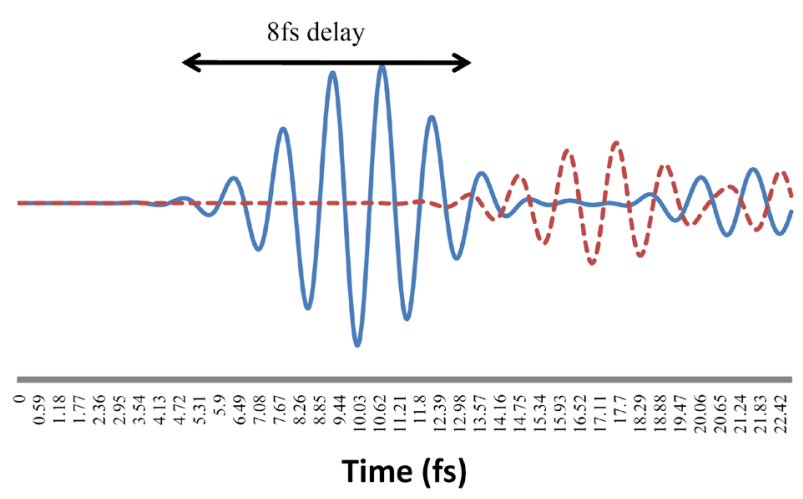

Figure 6. The temporal simulation of the switch shows a 8 fs delay in response.

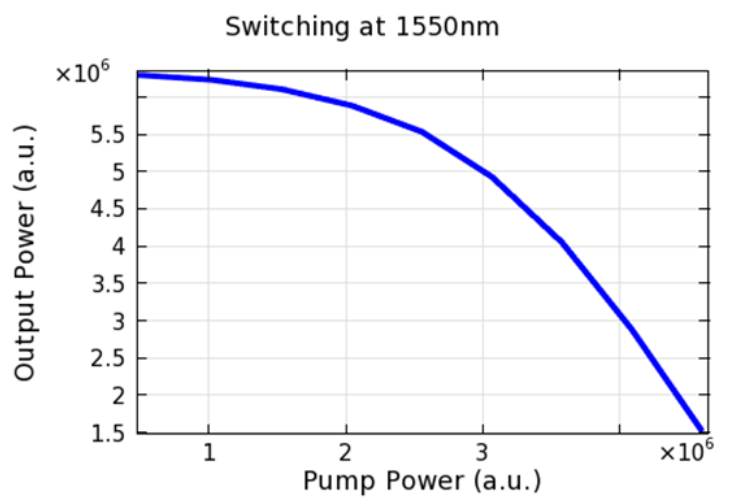

Figure 7. Dependence of Output power of the $1550 \mathrm{~nm}$ signal to power of the $1000 \mathrm{~nm}$ pump. 
dimension of the switch $(550 \mathrm{~nm})$ is the main interest in reduction of the loss. It means that this plasmonic device will be more suitable for designing high dense integrated optical devices. Using Finite Element Method (FEM) the operation of the switch is investigated. The overall length of the switch is $550 \mathrm{~nm}$ and extinction ratio is $-14.66 \mathrm{~dB}$.

\section{REFERENCES}

[1] R. Zia, J. A. Schuller, A. Chandran and M. L. Brongersma, "Plasmonics: The Next Chip-Scale Technology," Materials Today, Vol. 9, 2006, p. 20. http://dx.doi.org/10.1016/S1369-7021(06)71572-3

[2] D. K. Gramotnev and S. I. Bozhevolnyi, "Plasmonics beyond the Diffraction Limit," Nature Photonics, Vol. 4, 2010, pp. 83-91. http://dx.doi.org/10.1038/nphoton.2009.282

[3] W. L. Barnes, A. Dereux and T. W. Ebbesen, "Surface Plasmon Subwavelength Optics,” Nature, Vol. 424, 2003, pp. 824-830. http://dx.doi.org/10.1038/nature01937

[4] T. W. Ebbesen, C. Genet and S. I. Bozhevolnyi, "SurfacePlasmon Circuitry,” Physics Today, Vol. 61, 2008, pp. 44-50. http://dx.doi.org/10.1063/1.2930735

[5] S. A. Maier, "Plasmonics: The Promise of Highly Integrated Optical Devices,” IEEE Journal of Selected Topics in Quantum Electronics, Vol. 12, 2006, p. 1671. http://dx.doi.org/10.1109/JSTQE.2006.884086

[6] J. Park, H. Kim and B. Lee, "High Order Plasmonic Bragg Reflection in the Metal-Insulator-Metal Waveguide Bragg Grating,” Optics Express, Vol. 16, No. 1, 2008. http://dx.doi.org/10.1364/OE.16.000413

[7] Y. Chang, "Design and Analysis of Metal/Multi-Insulator/ Metal Waveguide Plasmonic Bragg grating," Optics Express, Vol. 18, No. 12, 2010. http://dx.doi.org/10.1364/OE.18.013258

[8] N. Nozhat and N. Granpayeh, "Switching Power Reduction in the Ultra-Compact Kerr Nonlinear Plasmonic Directional Coupler," Optics Communications, Vol. 285, 2012, pp. 1555-1559. http://dx.doi.org/10.1016/j.optcom.2011.11.110
[9] L. Sweatlock and K. Diest, "Vanadium Dioxide Based Plasmonic Modulators,” Optics Express, Vol. 20, No. 18, 2012.

[10] M. Pu, N. Yao, C. Hu, X. Xin, Z. Zhao, C. Wang and X. Luo, "Directional Coupler and Nonlinear Mach-Zehnder Interferometer Based on Metal-Insulator-Metal Plasmonic Waveguide," Optics Express, Vol. 18, No. 20, 2010, p. 21030. http://dx.doi.org/10.1364/OE.18.021030

[11] A. Y. Elezzabi, Z. Han, S. Sederberg and V. Van, "Ultrafast All-Optical Modulation in Silicon-Based Nanoplasmonic Devices," Optics Express, Vol. 17, No. 13, 2009, p. 11045,. http://dx.doi.org/10.1364/OE.17.011045

[12] S. Sederberg, D. Driedger, M. Nielsen and A. Y. Elezzabi, "Ultrafast All-Optical Switching in a Silicon-Based Plasmonic Nanoring Resonator," Optics Express, Vol. 19, No. 2, 2011.

[13] J. Liu, G. Fang, H. Zhao, Y. Zhang and S. Liu, "Surface Plasmon Reflector Based on Serial Stub Structure,” Optics Express, Vol. 17, No. 22, 2009. http://dx.doi.org/10.1364/OE.17.020134

[14] N. Zhu, T. Mei, “Analysis of an Ultra-Compact Wavelength Filter Based on Hybrid Plasmonic Waveguide Structure,” Optics Letters, Vol. 37, No. 10, 2012. http://dx.doi.org/10.1364/OL.37.001751

[15] J. Wei, J. Liu and M. Xiao, “ Giant Optical Nonlinearity of Silver-Doped Silicon Thin Film at Low Power Input: Laser-Triggered Cluster Resonance,” Applied Physics A, 2011, pp. 1031-1037. http://dx.doi.org/10.1007/s00339-011-6464-3

[16] A. Pannipitiya, I. D. Rukhlenko, M. Premaratne, H. T. Hattori and G. P. Agrawal, "Improved Transmission Model for Metal-Dielectric-Metal Plasmonic Waveguides with Stub Structure,” Optics Express, Vol. 18, No. 6, 2010. http://dx.doi.org/10.1364/OE.18.006191

[17] F. A. Jenkins and H. E. White, "Fundamentals of Optics," 4th Edition, McGraw-Hill, 1981.

[18] H. Kaatuzian, "Photonics,” Vol. 1, 2nd Edition, AUT Press, in Persian, 2008. 